\title{
NOTE ON THE END EFFECT IN THE ELECTROSTRICTION OF CYLINDRICAL CONDENSERS.
}

\author{
By Edwin C. Kemble.
}

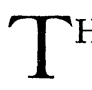

HERE are two methods of computing the deformations of bodies due to the action of electrostatic fields. One method substitutes the expressions for the ponderomotive body and surface forces derived in the general theory of electrostriction into the equations of elastic equilibrium and attempts to solve those equations subject to boundary conditions appropriate to the body under consideration. This will be called the "elasticity" method. The second mode of attack makes no use of the general theory but refers each particular problem back to fundamental principles. It assumes that the electrical and elastic state of the body can be defined with sufficient exactness by means of a finite number of coördinates. (For example, in the problem of the elongation of a charged cylindrical condenser, the coördinates chosen are the difference of potential between the coatings, $V$, and a longitudinal traction, $q$, to which the cylinder is supposed subjected.) Otherwise the assumptions of the two methods are the same. The second is essentially an energy method and will be referred to as such.

Hitherto the application of these two methods to the problem of the elongation of a charged cylindrical condenser has led to contradictory formulas. Sacerdote, ${ }^{1}$ using the energy method, derived a formula for the elongation of a condenser with adherent coatings in 1899 and one for the elongation of a condenser with nonadherent coatings in r9or. The first of these formulas did not seem to be in accord with the experimental results of More $^{2}$ and of Shearer. ${ }^{3}$ These experimenters found no elongation that could not be accounted for as the result of unavoidable heating, although the sensitiveness of their apparatus seemed sufficient to detect elongations of the order of magnitude of that predicted by the Sacerdote formula. This apparent disagreement between theory and experiment led Adams ${ }^{4}$ to attack the theoretical problem by the elasticity method. In I9I I he published formulas derived in this way which differed from the Sacerdote formulas and seemed to be in better accord

${ }^{1}$ Sacerdote, Journal de Phys. (3), t. VIII., p. 457, r899; t. X., p. 196, r9or.

${ }^{2}$ L. T. More, Phil. Mag. (5), I., p. I98, r900; (6), VI., p. I, I903; (6), X., p. 676, 1905.

${ }^{3}$ J. S. Shearer, Phys. Rev, (I), XIV., p. 89, Ig02.

${ }^{4}$ E. P. Adams, Phil. Mag. (6), XXII., p. 889, rgrr. 
with the experimental work. The present paper proposes to show that the formulas derived by the two different methods may be brought into agreement if the action of certain longitudinal forces due to the electric field in the neighborhood of the ends of the armature coatings be taken into account. ${ }^{1}$ It will also show that the negative results of More and of Shearer are not to be interpreted as a proof that there is no such thing as electrostriction.

The capacity of a cylindrical condenser of finite length is given approximately by the formula for the capacity of a section of an infinite cylindrical condenser having the same dimensions. To get the exact formula for the finite cylindrical condenser we must add to the approximate capacity an end correction independent of the length. This end correction becomes negligible in comparison with the whole capacity if the cylinder is very long. Reasoning by analogy one would be led to suppose that the end effect on the electrostriction would be negligible in the case of a long cylinder. But this is not the case. The longitudinal traction exerted by the electric field at the ends of the coatings is independent of the length of the coatings and consequently the elongation produced is proportional to that length.

We adopt the following notation:

$x, y, z$, Cartesian coördinates,

$r, \theta, z$, cylindrical coördinates,

\&, (Components $\left.\xi_{x}, \bigotimes_{y}, \xi_{z}\right)$ electromotive force,

$\mathfrak{D}$, (Components $\mathfrak{D}_{x}, \mathfrak{D}_{y}, \mathfrak{D}_{z}$ ) electric displacement,

$F$, (Components $F_{x}, F_{y}, F_{z}$ ) ponderomotive force per unit volume,

$f$, (Components $f_{x}, f_{y}, f_{z}$ ) ponderomotive force per unit area,

$K$, specific inductive capacity,

$E$, Young's modulus,

$\sigma$, Poisson's ratio,

$a$, internal radius of dielectric tube,

$b$, external radius of dielectric tube,

$d$, thickness of dielectric tube $(b-a)$,

$L$, length of coatings,

$V$, difference of potential between coatings,

$\rho$, volume density of electric charge,

$\delta_{1}, \delta_{2}$, constants characterizing the change in the specific inductive capacity of the dielectric when it is strained.

The general theory of electrostriction gives the following expression

1 Professor Adams has called my attention to the review of his article in the Beiblätter to the Annalen der Physik in which Pockels remarks that the discrepancy is probably due to the neglect of an end effect. So far as the present writer is aware this is the only published criticism of the work of Professor Adams. 
for the $x$-component of the ponderomotive force per unit volume in a dielectric which is isotropic at every point but not necessarily homogeneous:

$$
\begin{aligned}
F_{x}=\rho \epsilon_{x}-\frac{\epsilon^{2}}{8 \pi} \frac{\partial K}{\partial x} & -\frac{\mathrm{I}}{8 \pi}\left\{\frac { \partial } { \partial x } \left[\left(\xi_{x}^{2} \delta_{1}+\left(\xi_{y}^{2}+\xi_{z}^{2}\right) \delta_{2}\right]\right.\right. \\
+ & \frac{\partial}{\partial y}\left[\left(\xi_{x} \xi_{y}\left(\delta_{1}-\delta_{2}\right)\right]+\frac{\partial}{\partial z}\left[\xi_{x}\left(\xi_{z}\left(\delta_{1}-\delta_{2}\right)\right]\right\} .\right.
\end{aligned}
$$

The $y$ - and $z$-components may be obtained by cyclic advancement. In the case of an uncharged homogeneous dielectric the above reduces to the simple vector expression

$$
F=-\frac{\left(\delta_{1}+\delta_{2}\right)}{\mathrm{I} 6 \pi} \operatorname{grad}\left(\xi^{2}\right) .
$$

By means of (i) and its companion equations the expanded Maxwell "fictitious" stress system

$P_{x x}=\frac{K}{8 \pi}\left(\xi_{x}^{2}-\mathfrak{\xi}_{y}^{2}-\xi_{z}^{2}\right)-\frac{\mathrm{I}}{8 \pi}\left(\mathfrak{\xi}_{x}^{2} \delta_{1}+\mathfrak{F}_{y}^{2} \delta_{2}+\mathfrak{\xi}_{z}^{2} \delta_{2}\right)$,

$P_{y z}=\frac{K}{4 \pi} \xi_{y}\left(\xi_{z}+\frac{\mathrm{I}}{8 \pi}\left(\delta_{2}-\delta_{1}\right) \xi_{y} \xi_{z}\right.$

may be obtained. The expressions for the force exerted at a surface of discontinuity may be obtained either from (I) directly or from (3). Suppose that the $x$-axis coincides with the normal drawn from the medium $A$ to the medium $B$. Then the components of the force per unit area exerted at the surface are

$$
\begin{aligned}
f_{x} & =2 \pi \mathfrak{D}_{x}{ }^{2}\left(\frac{\mathrm{I}}{K^{\prime \prime}}-\frac{\mathrm{I}}{K^{\prime}}-\frac{\delta_{1}{ }^{\prime \prime}}{K^{\prime \prime 2}}+\frac{\delta_{1}{ }^{\prime}}{K^{\prime 2}}\right)-\frac{\left(\xi_{y}{ }^{2}+\left(\xi_{z}{ }^{2}\right)\right.}{8 \pi}\left(K^{\prime \prime}-K^{\prime}+\delta_{2}{ }^{\prime \prime}-\delta_{2}{ }^{\prime}\right), \\
f_{y} & =\frac{\mathfrak{\xi}_{y} \mathfrak{D}_{x}}{2}\left(\frac{\delta_{2}{ }^{\prime \prime}-\delta_{1}{ }^{\prime \prime}}{K^{\prime \prime}}-\frac{\delta_{2}{ }^{\prime}-\delta_{1}{ }^{\prime}}{K^{\prime}}\right), \\
f_{z} & =\frac{\mathfrak{\xi}_{z} \mathfrak{D}_{x}}{2}\left(\frac{\delta_{2}^{\prime \prime}-\delta_{1}^{\prime \prime}}{K^{\prime \prime}}-\frac{\delta_{2}{ }^{\prime \prime}-\delta_{1}{ }^{\prime}}{K^{\prime}}\right),
\end{aligned}
$$

where primes refer to the medium $A$ and double primes to medium $B$.

1 This is a specialization of the formulas given by Pockels, Encyk. der Math. Wiss., Band $\mathrm{V}_{2}$, Heft 2, pp. 354 and 360 .

2 Pockels, 1. c., p. 36 r

3 Pockels, 1. c., equations (5) and ( $\left.\mathrm{II}^{\prime}\right)$.

${ }^{4}$ Pockels, l. c., equation (13). 
Adherent Armatures.

Now consider the case of a cylindrical condenser with adherent armatures. Let the $z$-axis coincide with the axis of the cylinder. Then the electric force at points far from the ends of the coatings is

$$
\mathfrak{E}_{0}=\frac{V}{r} \frac{\mathrm{I}}{\log b / a} \equiv \frac{S}{r} .
$$

Substituting this value of $\&$ into $(2)$ we see that the ponderomotive force is radial and has the value

$$
F_{r}=\frac{\left(\delta_{1}+\delta_{2}\right)}{8 \pi} \frac{S^{2}}{r^{3}} .
$$

The surface forces are normal to the coatings and have the values

$$
\begin{aligned}
& f_{r} \text { (outer surface) }=-\frac{\left(K-\delta_{1}\right)}{8 \pi b^{2}} S^{2}, \\
& f_{r}(\text { inner surface })=+\frac{\left(K-\delta_{1}\right)}{8 \pi a^{2}} S^{2} .
\end{aligned}
$$

Professor Adams assumes that the longitudinal stress is zero for an open tube and computes the elongation due to the forces specified on the assumption that the cylinder is thin. He obtains

$$
\Delta_{1} L=-\left(\frac{V}{d}\right)^{2} \frac{\left(\delta_{1}+\delta_{2}\right) \sigma L}{8 \pi E} .
$$

The formula of Sacerdote is

$$
\Delta L=\left(\frac{V}{d}\right)^{2} \frac{\left[K+\delta_{2}-\left(\delta_{1}+\delta_{2}\right) \sigma\right] L}{8 \pi E} .
$$

Both Adams and Sacerdote assume that the armature coatings are so thin that their tensile strength may be neglected.

Let us now compute the electrical ponderomotive forces exerted at the ends of the coatings and add the elongation which they produce to that already computed on the assumption that there is no longitudinal traction.

There are three possible sources of longitudinal stress.

(a) At the edges of the coatings there is a strong electric force directed along the axis of the cylinder. The intensity of this force is evidenced by the brush discharge which frequently takes place at the edges of the coatings when they are charged to high potentials. This strong electric

1 E. P. Adams, Phil. Mag., 6, XXII., p. 892, equation (I2), neglecting $\left(\frac{d}{2 a}\right)\left(\frac{\mathrm{I}}{\mathrm{I}-\sigma}\right)$ in comparison with unity.

2 E. P. Adams, 1. c., equation (15); Pockels, 1. c., equation (23). 
field is accompanied by a mechanical force tending to stretch the coatings. This force is applied in the first place to the coatings but the stress is at once transmitted by them to the cylinder of dielectric.

(b) At points far from the ends of the cylinder the electric force in the dielectric is a function of $r$ only, so that the body force, $-\frac{\left(\delta_{1}+\delta_{2}\right)}{16 \pi} \operatorname{grad~} \aleph^{2}$, has no component along the z-axis. But near the edges of the coatings the value of $\xi^{2}$ begins to diminish and drops nearly to zero a short distance beyond the ends of the armatures. In this region the force

$$
F_{z}=-\frac{\left(\delta_{1}+\delta_{2}\right)}{\mathrm{I} 6 \pi} \frac{\partial\left(\xi^{2}\right)}{\partial z}
$$

must be taken into account.

(c) The third source of longitudinal traction is the z-component of the force on that part of the surface of the cylinder which is not covered by the armature coatings. (Where the coating is in contact with the surface of the dielectric the ponderomotive surface force has no tangential component.)

For our present purpose it is not necessary to compute these forces

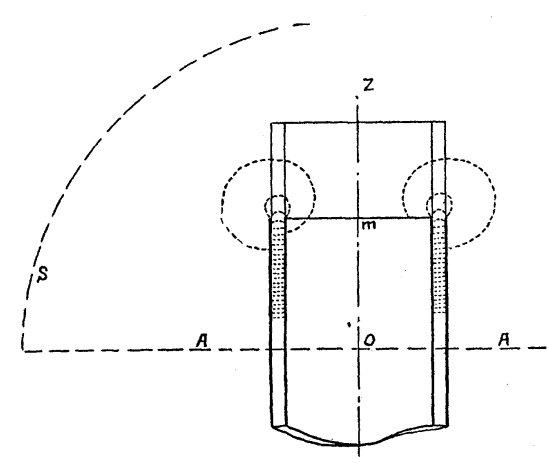

Fig. 1. separately. The resultant of all three may be obtained very simply from the expanded Maxwell stress system. The fundamental property of this system is that the surface integral of the stress over any closed surface is equal to the resultant ponderomotive force exerted on the material inside the surface. Consequently the total longitudinal pull exerted by the electric field in the neighborhood of the ends of the coatings may be obtained by integrating the $z$-component of the Maxwell stresses over a surface which encloses one end of the coatings.

To make the computation as simple as possible we choose as our surface, $S$ (Fig. I), a hemisphere with its base plane $A-A$ normal to the axis of the cylinder and cutting that axis at a point far from either end of the armatures. In the limit when the radius of the hemisphere increases indefinitely the integral of the Maxwell stress over $S$ approaches the integral of the Maxwell stress over the plane $A-A$. Let $S_{1}$ denote the annular section in which the plane $A-A$ cuts the cylinder. Let $S_{2}$ denote the rest of the plane $A-A$. As the distance $\overline{o m}$ from the plane 
$A-A$ to the end of the coatings is indefinitely increased the integral of the Maxwell stress over $S_{2}$ will approach zero as a limit. Let $P$ denote the longitudinal traction to be computed. Then to a close approximation $P$ is equal to the integral of the $z$-component of the Maxwell stress over $S_{1}$ only.

$$
P=\iint_{S_{1}} P_{z z} d s=\iint_{S_{1}} \frac{\mathfrak{F}^{2}\left(K+\delta_{2}\right)}{8 \pi} d s=\frac{K+\delta_{2}}{4} \int_{a}^{b} \xi^{2} r d r .
$$

But

Hence

$$
\aleph^{2}=\left(\frac{V}{r}\right)^{2}\left(\frac{\mathrm{r}}{\log b / a}\right)^{2}
$$

$$
P=\frac{V^{2}\left(K+\delta_{2}\right)}{4(\log b / a)^{2}} \int_{a}^{b} \frac{d r}{r}=\frac{V^{2}\left(K+\delta_{2}\right)}{4 \log b / a} .
$$

Now our preliminary analysis of the sources of this traction shows that the electrical field exerts no longitudinal pull except near the ends of the coatings. Hence we can obtain the elongation produced to a very close approximation if we treat $P$ as a force applied directly to the ends of the coatings. Then

$$
\frac{\Delta_{2} L}{L}=\frac{P}{\mathrm{E} \pi\left(b^{2}-a^{2}\right)}=\frac{V^{2}\left(K+\delta_{2}\right)}{4 \pi E\left(b^{2}-a^{2}\right) \log b / a} .
$$

If $(b-a)$ is small we have approximately

Hence

$$
\text { . }\left(b^{2}-a^{2}\right) \log b / a=2 d^{2} .
$$

$$
\frac{\Delta_{2} L}{L}=\frac{\left(\dot{K}+\delta_{2}\right)}{8 \pi E}\left(\frac{V}{d}\right)^{2} .
$$

Adding this elongation to that obtained by Professor Adams we have

$$
\frac{\Delta L}{L}=\frac{\Delta_{1} L}{L}+\frac{\Delta_{2} L}{L}=\frac{\mathrm{I}}{8 \pi E}\left(\frac{V}{d}\right)^{2}\left[K+\delta_{2}-\left(\delta_{1}+\delta_{2}\right) \sigma\right],
$$

which is the formula given by the energy method.

\section{Non-Adherent Armatures.}

Let us now suppose that the armatures are not in contact with the cylinder of dielectric but are separated from it by a nonconducting intermediary fluid of dielectric constant $K^{\prime}$. Let $g$ and $f$ denote the radii of the outer and inner armatures respectively. The electric force at points in the interior of the dielectric and far from the ends of the condenser will be sensibly uniform. Denote its value by $H$. 
The formula given by Sacerdote for this case is

$$
\frac{\Delta L}{L}=\frac{H^{2}}{8 \pi E}\left[\delta_{2}-\left(\delta_{1}+\delta_{2}\right) \sigma\right] .
$$

Professor Adams obtains

$$
\frac{\Delta_{1} L}{L}=-\frac{H^{2}}{8 \pi E}\left(\delta_{1}+\delta_{2}\right) \sigma .
$$

The discrepancy is

$$
\frac{\Delta_{2} L}{L}=\frac{\Delta L}{L}-\frac{\Delta_{1} L}{L}=\frac{\delta_{2} H^{2}}{8 \pi E} .
$$

This case differs from the previous one in that the longitudinal pull on the edges of the armatures is no longer transmitted to the cylinder, but is born by the armature itself. The force on the end of the tube can no longer be computed by means of the fictitious stresses but must be obtained by the separate calculation of the resultant $z$-components of the body-force and the surface force which act directly on the dielectric.

In what follows it will be assumed that the cylinder of dielectric extends beyond the edges of the armatures far enough so that the stray electric field at the end of the tube is of negligible intensity. Otherwise the longitudinal traction would depend on the length of the tube.

We choose the axis of the condenser as the z-axis and the plane $A-A$ as the $x y$-plane. Denote the value of the electric force in the $x y$-plane by $\mathbb{E}_{0}$.

The body force is easily computed. It is

$$
P^{\prime}=-\frac{\left(\delta_{1}+\delta_{2}\right)}{\mathrm{I} 6 \pi} \iiint \frac{\partial\left(\xi^{2}\right)}{\partial z} d v
$$

(Cf. equation (2).) The integral may be extended over the entire end of the tube although $\partial\left(\mathbb{F}^{2}\right) / \partial z$ is sensibly equal to zero except near the ends of the armatures. Let $L$ be the distance from the plane $A-A$ to the end of the cylinder. Inserting the limits of integration we have

$$
\begin{aligned}
& P^{\prime}=-\frac{\left(\delta_{1}+\delta_{2}\right)}{\mathrm{I} 6 \pi} \int_{0}^{2 \pi} d \theta \int_{a}^{b} r d r \int_{0}^{L} \frac{\partial\left(\xi^{2}\right)}{\partial z} d z
\end{aligned}
$$

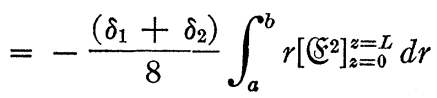

$$
\begin{aligned}
& =\frac{\left(\delta_{1}+\delta_{2}\right)}{8} \int_{a}^{b} r \mathbb{E}_{0}^{2} d r .
\end{aligned}
$$

The surface force will now be computed. Quantities referring to the

${ }^{1}$ Sacerdote, J. de Phys. [3], XI., p. 196, r9or.

2 Adams, 1. c., equation 19. 
inner and outer surfaces will be denoted by primes and double primes respectively. Let

For a fluid

$$
\mathfrak{D}_{x}^{2}+\mathfrak{D}_{y}^{2}=\mathfrak{D}_{r}^{2} \text {. }
$$

$$
\delta_{1}=\delta_{2} \text {. }
$$

Consequently the expressions for the $z$-components of the forces per unit area on the inner and outer surfaces become

$$
f_{z}^{\prime}=\frac{\mathfrak{E}_{z}^{\prime} \mathfrak{D}_{r}^{\prime}}{2}\left[\frac{\delta_{2}-\delta_{1}}{K}\right]
$$

and

$$
f_{z}^{\prime \prime}=-\frac{\xi_{z}^{\prime \prime}}{2} \frac{\mathfrak{D}_{r}^{\prime \prime}}{2}\left[\frac{\delta_{2}-\delta_{1}}{K}\right] \text {. }
$$

Let $\mathfrak{F}_{r}{ }^{\prime}$ and $\mathfrak{F}_{r}{ }^{\prime \prime}$ denote the radical components of the electric force in the tube of dielectric at its inner and outer surfaces respectively. Then

$$
\begin{aligned}
& f_{z}^{\prime}=\left(\frac{\delta_{2}-\delta_{1}}{8 \pi}\right) \xi_{z}^{\prime} \xi_{r}^{\prime}, \\
& f_{z}^{\prime \prime}=-\left(\frac{\delta_{2}-\delta_{1}}{8 \pi}\right) \mathbb{E}_{z}{ }^{\prime \prime} छ_{r}^{\prime \prime} .
\end{aligned}
$$

The total traction exerted on the surface is

$$
\begin{aligned}
& P^{\prime \prime}=\int_{0}^{2 \pi} d \theta \int_{0}^{L} a f_{z}^{\prime} d z+\int_{0}^{2 \pi} d \theta \int_{0}^{L} b f_{z}^{\prime \prime} d z \\
& =\frac{\delta_{2}-\delta_{1}}{8 \pi} \int_{0}^{L} d z \int_{0}^{2 \pi} d \theta\left[a \mathfrak{E}_{z}^{\prime} \mathfrak{E}_{r}^{\prime}-b \mathfrak{F}_{z}^{\prime \prime} \mathfrak{জ}_{r}^{\prime \prime}\right] \\
& =-\frac{\delta_{2}-\delta_{1}}{\delta \pi} \int_{0}^{L} d z \int_{0}^{2 \pi} d \theta \int_{a}^{b} \frac{\partial\left(r \xi_{z} \xi_{r}\right)}{\partial r} d r .
\end{aligned}
$$

Both the curl and the divergence of the electric force vanish in the interior of the dielectric. Hence

Therefore

$$
\frac{\partial\left(r \mathbb{E}_{z} \mathfrak{E}_{r}\right)}{\partial r}=r \mathbb{E}_{r} \frac{\partial \mathfrak{E}_{r}}{\partial z}-r \mathbb{E}_{z} \frac{\partial \mathfrak{E}_{z}}{\partial z}=\frac{r}{2} \frac{\partial}{\partial z}\left[\mathfrak{F}_{r}^{2}-\mathfrak{E}_{z}^{2}\right] .
$$

$$
P^{\prime \prime}=\frac{\delta_{1}-\delta_{2}}{\mathrm{I} 6 \pi} \iiint r \frac{\partial}{\partial z}\left(\xi_{r}^{2}-\xi_{z}^{2}\right) d r d \theta d z .
$$

If we integrate with respect to $z$ and $\theta$, remembering that $\left(\mathscr{F}_{\mathbf{r}}{ }^{2}-\mathscr{E}_{z}{ }^{2}\right)$ vanishes at $z=L$ and reduces to $\varepsilon_{0}^{2}$ at $z=0$, we obtain

$$
P^{\prime \prime}=\frac{\delta_{2}-\delta_{1}}{8} \int_{a}^{b} r \mathfrak{E}_{0}^{2} d r \text {. }
$$

1 Cf. equations (4). 
The total longitudinal traction is

$$
P=P^{\prime}+P^{\prime \prime}=\frac{\delta_{2}}{4} \int_{a}^{b} r \varepsilon_{0}^{2} d r
$$

or, if the variation of $\epsilon_{0}$ with $r$ be neglected

$$
P=\frac{a d \delta_{2} H^{2}}{4} .
$$

The corresponding elongation is

$$
\frac{\Delta^{\prime} L}{L}=\frac{P}{2 \pi a d E}=\frac{\delta_{2} H^{2}}{8 \pi E}
$$

This is the discrepancy we have already found between the formulas of Adams and Sacerdote.

\section{Liquid Armatures.}

In our discussion of condensers having adherent armatures we have tacitly assumed that these armatures are thin metal sheets. It has been a common practice on the part of experimenters in this field to use a conducting liquid for one of the armatures. That we can use the same formula for this case as for that in which both armatures are of metal foil is not immediately obvious. In fact we can show that if both armatures are liquid in contact with the dielectric cylinder the elongation is the same as if the armatures were nonadherent.

In the case of the typical adherent armatures first discussed the longitudinal traction is

$$
P=\frac{K+\delta_{2}}{4} \int_{a}^{b} \xi_{0}^{2} r d r
$$

The longitudinal pull exerted directly on the tube is

$$
P_{1}=\frac{\delta_{2}}{4} \int_{a}^{b} \mathfrak{F}_{0}^{2} r d r
$$

Consequently the traction exerted on the edges of the armatures must be

$$
P_{2}=\frac{K}{4} \int_{a}^{b} \mathfrak{E}_{0}^{2} r d r
$$

(This could also be proved directly.) Now in the case of liquid armatures this force is not transmitted to the tube but is borne by the liquid itself. Consequently we may neglect $P_{2}$ in computing the elongation of the tube. In this way we get the same formula as for the case of nonadherent armatures.

If the two armatures are of the same length we may assume that each carries half of the traction $P_{2}$. Then if one of them is a conducting liquid we may compute the elongation from (9) if we substitute $K / 2$ for $K$. 
However, the usual experimental arrangement is to have the liquid armature very much longer than the coating of metal foil. With this arrangement the two armatures do not carry equal longitudinal tractions. In fact there is no longitudinal pull on the liquid dielectric at all, for the lines of force arrange themselves as indicated in Fig. 2, and the metal

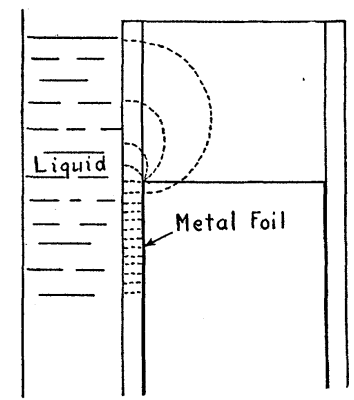

Fig. 2.

foil must carry the whole of the traction $P_{2}$. Consequently the whole stress is transmitted to the dielectric and the elongation is the same as if both armatures were metal sheets.

\section{The Experiments of More and of Shearer.}

The above work shows that if the general theory of electrostriction is correct the experimental results of More and of Shearer must be reconciled with the Sacerdote formula, (9). Moreover, it shows that the principal term of (9), $(K L / 8 \pi E)(V / d)^{2}$, is not due to a direct action of the electric field on the dielectric but to the longitudinal pull on the edges of the armatures. If there were no direct electrostrictive action (as has been suggested) we would still expect an elongation given by the formula

$$
\frac{\Delta L}{L}=\frac{K}{8 \pi E}\left(\frac{V}{d}\right)^{2} .
$$

Since the publication of the work of More and of Shearer, Professor Adams $^{1}$ has computed the value of the combination of constants $\left[\delta_{1}-\left(\delta_{1}+\delta_{2}\right) \sigma\right]$ for several different kinds of glass from the experiments of Wüllner and Wien on the change in the capacity of cylindrical glass condensers when they are stretched. The computed values vary from +0.68 to -2.85 . For glasses for which this combination of constants has a negative value the formula (9) predicts a smaller elongation than does (29). Hence the experimental results of More and of Shearer

${ }^{1}$ E. P. Adams, 1. c., p. 895 . 
are more easily explained if the general theory of electrostriction is assumed to be correct than otherwise.

The elongation predicted for tube No. 5 of Professor More's second series of experiments ${ }^{1}$ by (9) is $3.6 \times \mathrm{IO}^{-6} \mathrm{~cm}$. or 6 divisions of Professor More's micrometer scale if the extreme value -2.85 of the combination of constants $\left[\delta_{2}+\left(\delta_{1}+\delta_{2}\right) \sigma\right]$ is used. The smallest elongations observed for this tube were 5 and 7 micrometer divisions. Possibly an appreciable fraction of these minimum observed elongations was due to heat but the discrepancy between theory and experiment can hardly be considered to amount to a contradiction.

JefFerson Physical Laboratory,

Harvard University.

${ }^{1}$ L. T. More, Phil. Mag. (6), 6, p. I3, I903. 